\title{
A Rare Form of Adult Onset Leukodystrophy: Orthochromatic Leukodystrophy with Pigmented Glia
}

\author{
P. Shannon, J.R. Wherrett and S. Nag
}

\begin{abstract}
Background: Orthochromatic leukodystrophy with pigmented glia and scavenger cells is a rare leukodystrophy of unknown etiology. This report describes a 42-year-old man with a history of depression, dementia and parkinsonism having the pathological features of orthochromatic leukodystrophy with pigmented glia. Methods: We reviewed the clinical history and pathology of autopsy and brain biopsy material. Results: Imaging revealed bilateral cerebral white matter hypodensities. At autopsy, the brain demonstrated a leukodystrophy affecting predominantly the cerebral hemispheres and characterized by demyelination, and cytoplasmic pigment deposits in oligodendroglia and astrocytes. The pigment had the staining properties of ceroid-lipofuschin and on ultrastructural examination was composed of membrane-bound lipid and electron-dense inclusions which had a fingerprint-like pattern. Similar pigment inclusions were not observed on ultrastructural examination of renal, splenic or hepatic tissue obtained at autopsy. The brain biopsy contained cerebral cortex with sparse subcortical white matter in which a few oligodendroglia and fewer astrocytes at the grey/white junctions showed cytoplasmic pigmentary inclusions identical to those described above. However, due to the paucity of white matter in the specimen a definite diagnosis of orthochromatic leukodystrophy with pigmented glia was not made. Conclusions: The diagnosis of orthochromatic leukodystrophy with pigmented glia and scavenger cells can only be made antemortem if the brain biopsy contains adequate white matter and although a rare condition, it should be considered in the differential diagnosis of an adult onset leukodystrophy.
\end{abstract}

RÉSUMÉ: Une forme adulte rare de leucodystrophie: la leucodystrophie orthochromatique avec névroglie pigmentée. Introduction: La leucodystrophie orthochromatique avec présence de névroglie pigmentée et de phagocytes est une leucodystrophie rare d'étiologie inconnue. Méthodes: Nous décrivons le cas d'un homme de 42 ans qui avait une histoire de dépression, de démence et de parkinsonisme ayant les caractéristiques anatomopathologiques de la leucodystrophie orthochromatique avec cellules pigmentées. Nous révisons l'histoire clinique et l'anatomopathologie des tissus prélevés à l'autopsie ainsi que du matériel obtenu par biopsie. Résultats: L'imagerie a révélé des zones hypodenses de la substance blanche dans les deux hémisphères. À l'autopsie, le cerveau présentait une leucodystrophie atteignant surtout les hémisphères et caractérisée par une démyélinisation et des dépôts cytoplasmiques de pigment dans l'oligodendroglie et dans les astrocytes. Le pigment avait les propriétés tinctoriales de la céroïd-lipofuscine et l'examen ultra structural a montré qu'il était composé de lipides liés à la membrane et d'inclusions opaques aux électrons qui avaient un motif d'empreinte digitale. Des inclusions de pigment similaires n’ont pas été observées à l'examen ultra structural de tissu rénal, splénique ou hépatique obtenu à l'autopsie. La biopsie du cerveau contenait du cortex cérébral avec peu de substance blanche dans laquelle quelques oligodendrocytes et peu d'astrocytes aux jonctions substance grise/substance blanche présentaient des inclusions cytoplasmiques pigmentaires identiques à celles décrites plus haut. Cependant, à cause de la rareté de la matière blanche dans le spécimen, un diagnostic définitif de leucodystrophie orthochromatique avec névroglie pigmentée n'a pas été posé. Conclusions: Le diagnostic de leucodystrophie orthochromatique avec névroglie pigmentée et phagocytes ne peut être fait avant le décès que si la biopsie du cerveau contient une quantité adéquate de substance blanche. Bien que cette maladie soit rare, elle devrait être considérée dans le diagnostic différentiel de la leucodystrophie de l'âge adulte.

Can. J. Neurol. Sci. 1997; 24: 146-150

The orthochromatic leukodystrophies describe a heterogenous group of diseases characterized by the presence of sudanophilic (orthochromatic) myelin breakdown products. Such orthochromatic material may be present in a wide variety of white matter disease, including infarcts, multiple sclerosis, and the majority of leukodystrophies. Peiffer ${ }^{1}$ identified a subset of orthochromatic leukodystrophies (OLD) which he termed "orthochromatic leukodystrophy with pigmented glia and scavenger cells". These cases showed prominent cerebral demyelination associated with glial and microglial cytoplasmic pigment inclusions with the staining properties of ceroid-lipofuscin. The specific features of other leukodystrophies (e.g., metachromatic leukodystrophy, Krabbe's disease, Alexander's disease, Canavan's disease, adrenoleukodystrophy) or demyelinating diseases were absent and the presence of pigmented glia separated this group from orthochromatic leukodystrophy of the simple type.

Since the first case report of this disease by Van Bogaert and Nyssen $^{2}$ in 1936 , approximately 25 cases of OLD with pig-

From the Department of Pathology (Division of Neuropathology) (P.S., S.N.) and Division of Neurology (J.R.W.), University of Toronto and The Toronto Hospital, Toronto, Ontario, Canada.

RECEIVED JUNE 24, 1996. ACCEPTED IN FINAL FORM NOVEMBER 15, 1996.

Reprint requests to: S. Nag, Division of Neuropathology, The Toronto Hospital, Western Division, 399 Bathurst Street, Toronto, Ontario, Canada M5T 2S8 
enigmatic, having a variable age of onset, a usually protracted clinical course, and antemortem diagnosis is uncommon. This case report describes a patient with OLD with pigmented glia and scavenger cells, with histological and ultrastructural examination of brain, liver, spleen and kidney.

\section{Case Report}

The patient, a 42-year-old man, was referred by a psychiatrist for neurological assessment of cognitive and motor symptoms. He had been depressed for 5 years, and had required 2 hospital admissions. He received electroconvulsive therapy on the second admission. At the time of neurologic consultation, he was receiving alprazolam, triazolam and desipramine. His wife described slowness of walking with falling, lack of energy and poor memory progressing over the time he had been depressed. His family had noticed a shaking in his hands. As well, there was increased frequency of urination, and a gain of $50 \mathrm{lbs}$ weight in 7 years. A brother with a known history of depression had committed suicide. The patient had four healthy children and had completed one year of high school.

On examination, he scored 24/30 on the Folstein Mini-Mental Status test. His affect was flat and there was diminished spontaneous blinking with normal facial expression. There was cogwheel rigidity at the wrists and impaired rate of alternating movements. The finger-nose test was done slowly and heel to shin test was mildly clumsy. Postural reflexes were impaired, and the gait was wide-based with reduced range of arm swing. A CT head scan showed mild dilatation of both lateral ventricles and diffuse hypodensity in the periventricular regions. Blood counts, sedimentation rate, electrolytes, blood glucose, renal, liver and thyroid function tests, serum vitamin $B_{12}$ and VDRL were normal.

On follow-up four months later he was unchanged, except that the signs of parkinsonism were less apparent. Five months after presentation, formal neuropsychiatric testing revealed a performance IQ of 68 and a verbal IQ of 70 . Mild cogwheel rigidity was again present. He was receiving trazodone. An EEG showed diffuse dysrhythmia. Visual, auditory and somatosensory evoked responses were reported to be normal. A RISA scan was normal. Spinal fluid protein was $0.58 \mathrm{~g} / \mathrm{L}$, without leukocytosis, and oligoclonal bands were absent on electrophoresis. Blood assays for arylsulfatase, $B$-galactosidase and $B$-hexosaminidase in blood samples were normal. Cystoscopy and urodynamic testing revealed prostatitis and a hyper-reflexic bladder, and treatment with trimethoprim/sulfamethoxazole was begun.

Eight months after presentation, he was noted to be emotionally labile, with a voracious appetite and without change in libido. Urinary frequency was no longer present. MR imaging showed extensive white matter changes bilaterally. Thirteen months after presentation he was admitted for brain biopsy. Examination at this time revealed a systolic ejection murmur and a left carotid bruit. Concentration and memory were impaired. Frontal release signs were present and there was perseveration of motor responses. Startle responses were not excessive. Mild ataxia of the left limbs and left-sided visual and tactile inattention were queried. EMG and nerve conduction studies were normal. Serum cortisols were also normal. When seen eighteen months after presentation, his condition was unchanged. The patient was lost to further neurological follow-up, and died six years and eight months after presentation. Systemic autopsy was normal with the exception of bronchopneumonia.

\section{Materials ANd Methods}

The brain biopsy and blocks of brain, spleen, liver and kidney obtained at autopsy were processed using standard techniques and tissues were embedded in paraffin for light microscopy. Sections were stained with hematoxylin and eosin, luxol fast blue, Perl's stain for iron, PAS diastase, FontanaMasson, Sudan Black, and modified Bielchowsky stains. The indirect streptavidin-biotin peroxidase method was used for immunohistochemical localization of neurofilament (DakoNF,2F11 mouse monoclonal antibody, 1:50 dilution) and glial fibrillary acid protein (DAKO, 1:200 dilution).
Samples of brain, spleen, kidney and liver were also fixed in glutaraldehyde for electron microscopy. Samples were postfixed with osmium tetroxide and processed using standard techniques. Blocks were embedded in Epon 812 and semithin sections were examined after staining with $1 \%$ toluidine blue. Thin sections were stained with uranyl acetate and lead citrate and examined with a Joel CX100 electron microscope at $60 \mathrm{kV}$.

\section{Pathology}

The brain weighed 1180 grams before fixation. The right hemibrain fixed in formalin was available for examination. External examination was unremarkable, with a normal gyral pattern. On coronal sectioning, there was marked shrinkage and $\tan$ /brownish discoloration of the telencephalic white matter with extensive cavitation in the periventricular region extending to the subcortical $U$ fibres which were spared. These changes showed a marked anterior/posterior gradient, and were most prominent in the frontal lobe (Figure 1a). The occipital lobe white matter showed only a tawny discoloration. White matter

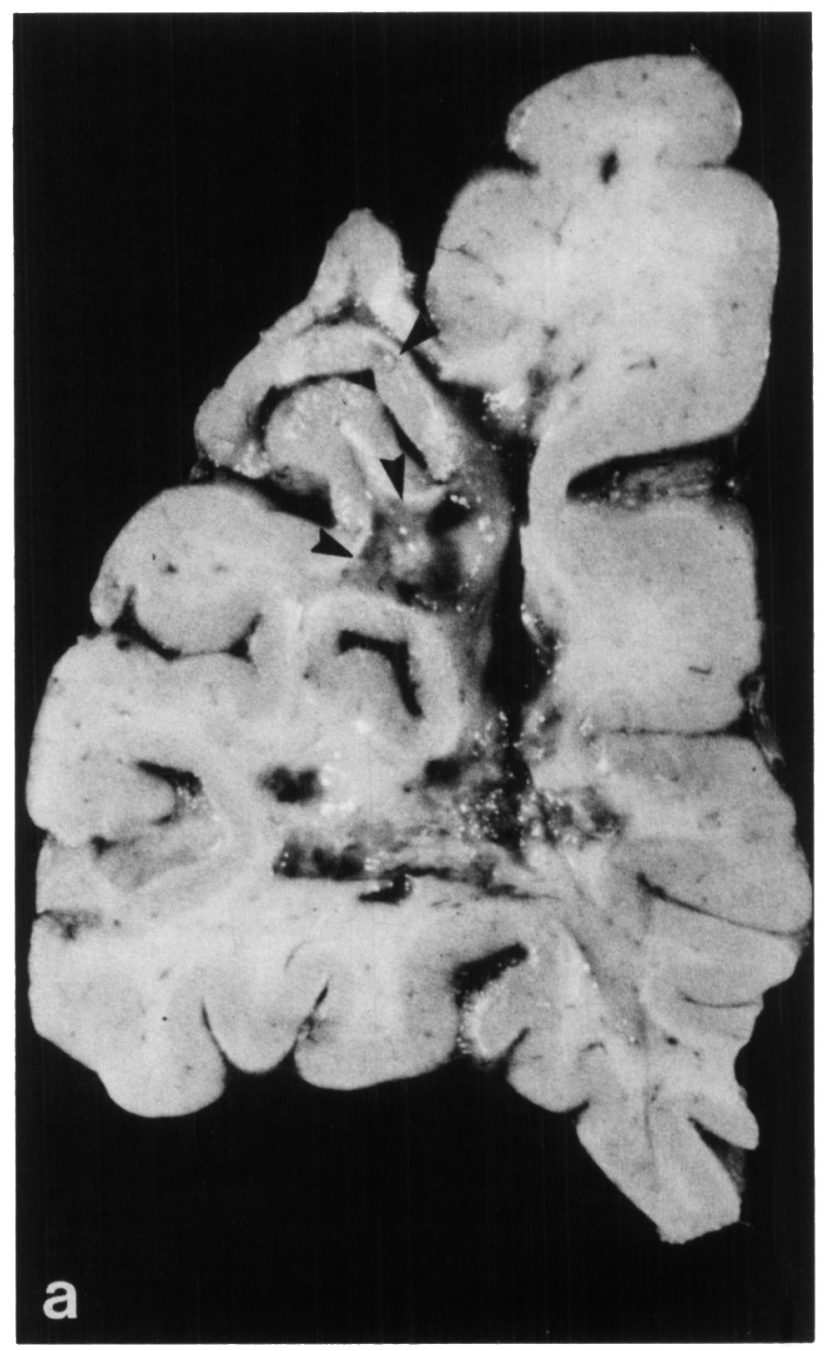

Figure 1: a) Coronal section of the right frontal lobe showing shrinkage and central cavitation of the white matter. Note preservation of the $U$ fibres (arrowheads). The cerebral cortex appears to be of normal thickness. b, c) Photomicrographs of the grey/white junction. 


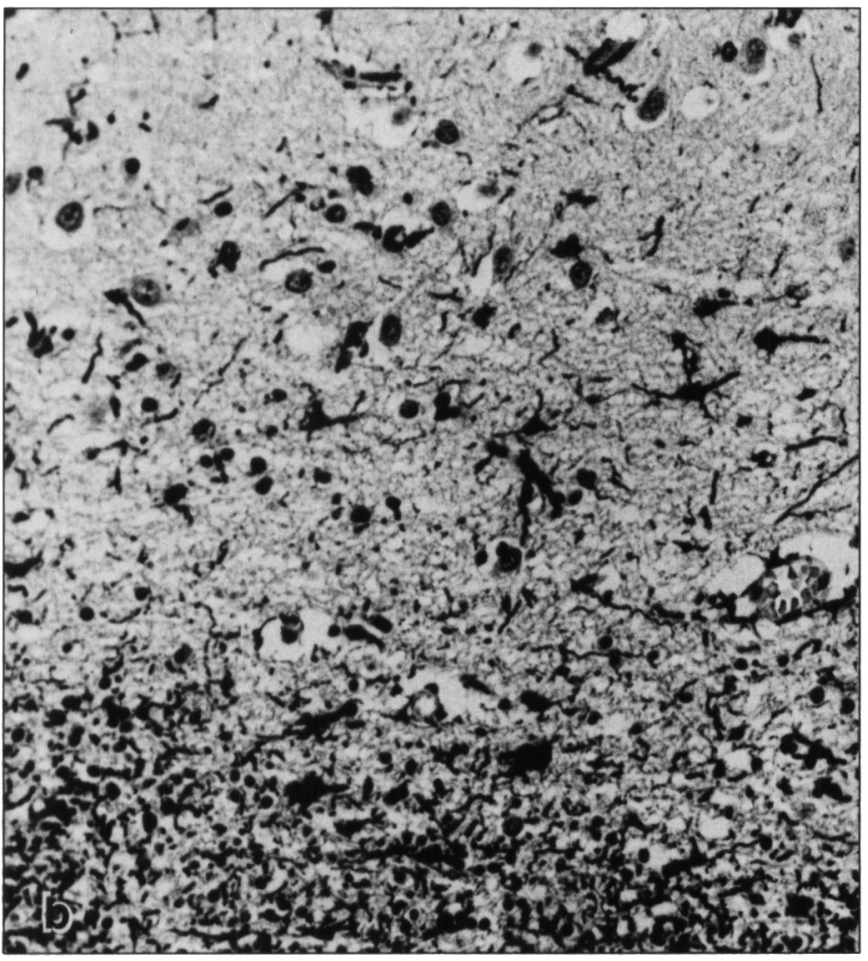

Figure 1: b) GFAP immunostaining highlights the moderate gliosis in the deep cortex and the marked gliosis in the underlying white matter. GFAP $\times 300$.

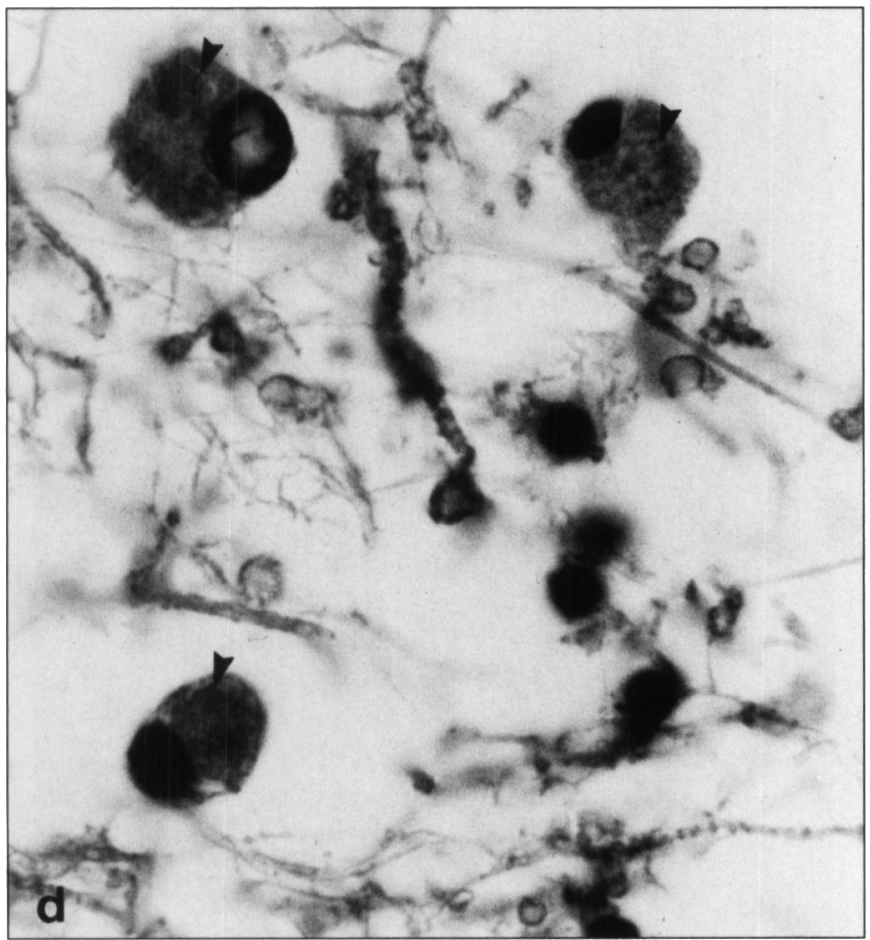

Figure 1: d) High magnification of the white matter shows three oligodendroglial cells containing cytoplasmic inclusions (arrowheads). $H \& E / L F B \times 1550$.

discoloration extended into the midbrain. The cerebellum, pons and medulla were grossly normal.

Histologic examination showed preservation of the cortical architecture with mild neuronal loss in laminae V and VI, asso-

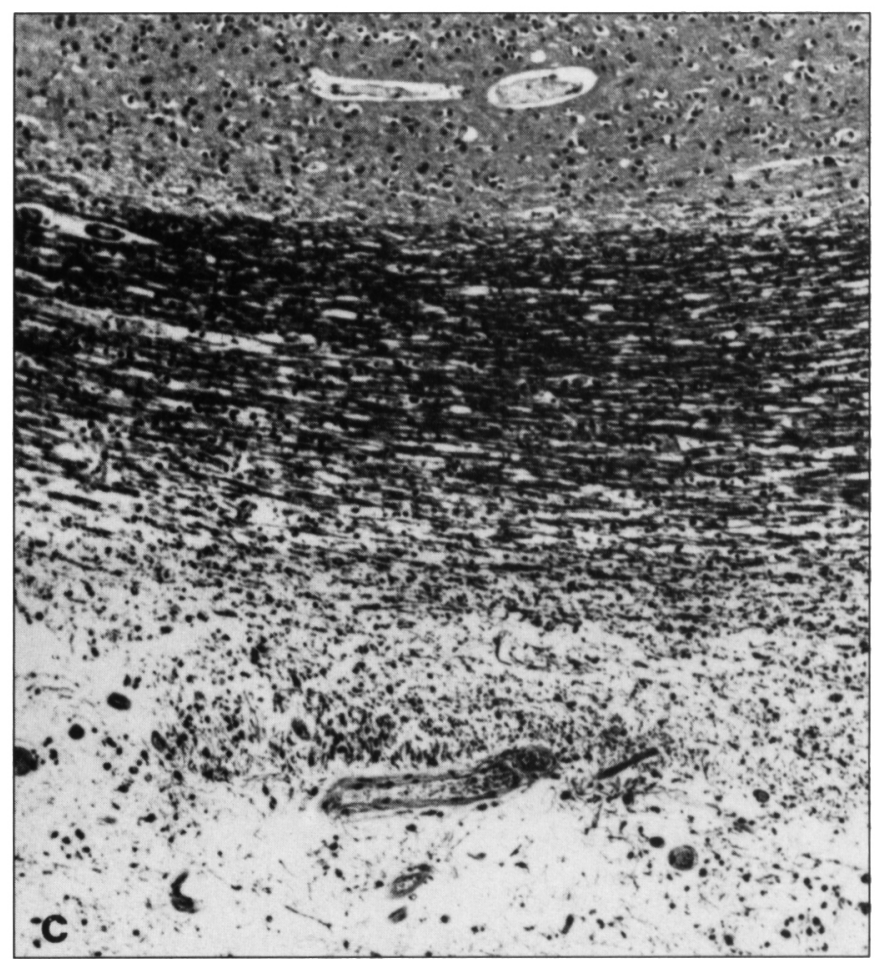

Figure 1:c) Severe loss of white matter and cavitation extending up to the $U$ fibres is present. $H \& E / L F B \times 150$.

ciated with a mild to moderate cortical gliosis (Figure 1b). Gliosis was also noted within the thalamus, striatum, and rostral midbrain nuclei. No abnormalities of the neuronal soma were seen; in particular there were no ballooned neurons or increase in cytoplasmic storage material and the cytoplasm contained normal amounts of lipofuschin pigment.

The white matter showed diffuse loss of myelin associated with an astrogliosis (Figure 1b). These changes were most marked in the frontal and periventricular region, with sparing of the subcortical $U$ fibres (Figure 1c). Although the temporal lobe was affected, the fornix and the optic tract were spared. Oligodendroglia and some astrocytes showed tan/golden-brown cytoplasmic inclusions on hematoxylin and eosin stained sections (Figure 1d). These inclusions showed yellow-green autofluorescence under UV light, and stained with Sudan Black and PAS diastase, rarely stained with Perl's reaction for iron, and stained inconstantly with the Fontana-Masson stain. Astrogliosis and pigmented glia were also present, although to a milder degree, in the white matter tracts of the midbrain, pons, cerebellum and medulla.

There were occasional small aggregates of perivascular macrophages most notably in the frontal white matter. These macrophages contained inclusions with a similar appearance to those in glia, but the material inside macrophages stained positively for iron as well. No globoid cells were seen, and there were no lymphoid infiltrates.

Axonal loss was observed adjacent to the areas of cavitary change; however, the degree of myelin loss was out of proportion to axonal loss, and sections stained with anti-neurofilament antibody and counterstained with luxol fast blue revealed numerous naked axons. Axonal swellings were also present, but were generally small (on the order of 10 microns in diameter) 
and were most common in the white matter of the frontal lobe, putamen and medulla.

Histological examination of the brain biopsy showed mainly cerebral cortex, with sparse amounts of white matter. There was mild cortical gliosis and pigmented oligodendroglia and astrocytes were present at the grey/white junctions. The staining properties of the glial pigment inclusions were the same as seen in the autopsy specimen.

Semi-thin sections of plastic-embedded material did not contain metachromatic material when stained with toluidine blue. Ultrastructural examination of both the frontal lobe biopsy and of brain obtained at autopsy showed membrane-bound deposits of electron-dense material, usually in association with lipid, within both oligodendroglia and astrocytes (Figure 2a). The electron-dense material was composed in part of amorphous granular material which was almost always associated with fingerprint-like profiles. The fingerprint-like pattern consisted of alternate layers of electron-dense and electronlucent material with an approximate periodicity of $5 \mathrm{~nm}$, arranged in short curved stacks or small whorls (Figure 2b).

Extensive ultrastructural examination of hepatic, renal and splenic tissue showed lipofuschin pigment in appropriate amounts within hepatocytes, Kupffer cells, splenic macrophages and renal tubular epithelial cells; however no fingerprint-like deposits were identified.

\section{Discussion}

Orthochromatic leukodystrophy (OLD) with pigmented glia and scavenger cells have a wide range of clinical presentations, familial patterns of occurrence, anatomical patterns of disease and histochemical findings. As in this patient, extrapyramidal motor symptoms including cogwheel rigidity ${ }^{3}$ or cerebellar ataxia $^{4,5}$ may be prominent early on, although pyramidal tract syndromes may be more frequent. ${ }^{6,7}$ The age of onset is variable; the earliest symptomatic age is at birth ${ }^{4}$ while other patients were not symptomatic until the fifth decade of life. ${ }^{1}$ Most do not present before the third decade.

The pattern of inheritance also seems variable. Autosomal dominant inheritance is likely in at least one kindred, ${ }^{6}$ and autosomal recessive inheritance in others. ${ }^{9}$ However, it is not entirely

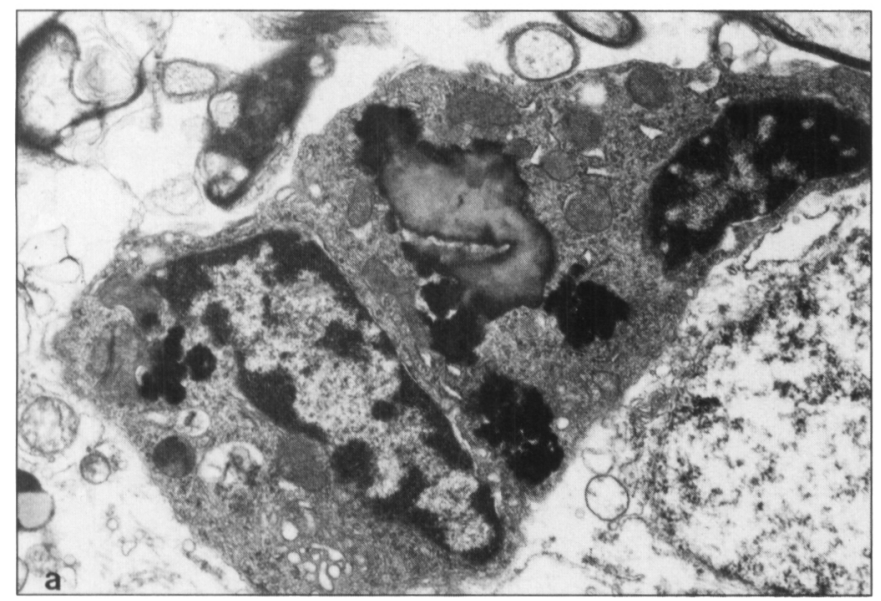

Figure 2: a) Electron micrograph showing membrane-bound, electrondense intracytoplasmic inclusions in two oligodendrocytes, $x$ 19,700.

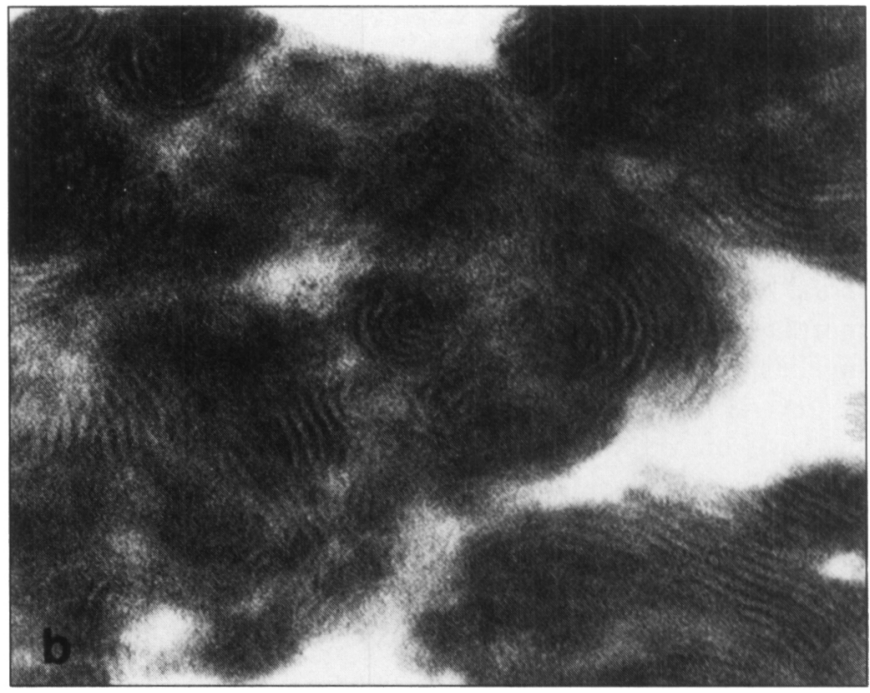

Figure 2: b) On higher magnification these inclusions have a fingerprint-like pattern consisting of alternating layers of electron-dense and electronlucent material with an approximate periodicity of $5 \mathrm{~nm} . x$ 188,000 .

clear whether some of the cases labeled as autosomal recessive, particularly in the older literature, might conceivably be due to an autosomal dominant trait with variable penetrance. Furthermore, the presence or absence of mood disorders among relatives is not mentioned in some case reports of apparently sporadic disease 4 and may be relevant. As in this case, psychiatric symptoms may predominate early in the course of the disease $^{5-7,10}$ and in the present case a family history of suicide is highly suggestive of a familial disorder.

In familial cases, the gross picture of demyelinating disease containing occasional axonal swellings with a slight predilection for the frontal lobes is well documented ${ }^{5,7}$ as is the presence of brown or tawny discoloration of the cerebral white matter with gliosis but not demyelination of the brainstem tracts. Most reports emphasize the predominantly telencephalic demyelination which, as in this case, relatively spares the optic radiation. In contrast to this case extensive cerebellar white matter disease has been reported ${ }^{5}$ as has Purkinje cell loss. ${ }^{4}$

Although myelin loss may be severe, the white matter cavitation observed in this case is unusual for this disease; ${ }^{1}$ although it is well documented in simple OLD, but the presence of pigmented glia in this case rules out the latter diagnosis. The presence of cortical gliosis in the face of mild neuronal depletion and absence of reactive changes in the cell bodies suggests that the white matter injury is a very slowly evolving process.

The presence of pigmented glia is necessary but not specific for diagnosis of this leukodystrophy. The glial inclusions are generally agreed to contain lipid and proteolipid, and to have the general staining properties of ceroid-lipofuschin. ${ }^{1,7}$ However, within a single case, the degree of PAS positivity and amount of stainable iron has been noted to vary. ${ }^{7}$ In the present case, the Fontana-Masson reaction did not always reliably stain the pigment deposits. Given that histochemical stains are occasionally capricious, autofluorescence is a reliable indicator of the presence of lipofuscin. Pigment is also readily seen and localized reliably to cell type in plastic embedded semi-thin sections stained with toluidine blue. 
Pigmented glia may occur wherever there is extensive myelin breakdown ${ }^{11}$ but are not an inevitable accompaniment to sudanophilic leukodystrophy. ${ }^{1}$ Similarly, the presence of fingerprint-like profiles within electron-dense, membrane bound material is non-specific, having been reported in cases of multiple sclerosis, post-traumatic white matter disease ${ }^{11}$ and progressive multifocal leukoencephalopathy. ${ }^{12}$ The presence of these bodies in the absence of a history of trauma or in the absence of other morphologic features of well-defined demyelinating or dysmyelinating diseases has not been reported.

Several attempts have been made to diagnose this entity on the basis of peripheral nerve biopsy ${ }^{5,7}$ but have not been successful in identifying diagnostic abnormalities. In this patient, ultrastructural examination of multiple extraneural visceral organs failed to reveal fingerprint-like profiles. The absence of such inclusions in extraneural visceral organs has not been previously documented and supports the contention that the inclusions are a product of myelin breakdown.

The brain biopsy in this case was not diagnostic, as insufficient white matter was present for assessment. Although the typical histologic and ultrastructural features of the pigment were present, these inclusions are non-specific and it would seem prudent to rule out acquired white matter disease after examination of a substantial portion of white matter. In this respect, the case reported by Calandriello et al. ${ }^{13}$ is interesting. In this paper, the authors entertain a diagnosis of OLD with pigmented glia on the basis of an appropriate history, clinical investigation and brain biopsy. Although the biopsy showed sudanophilic material within glia and microglia, no ceroid-lipofuschin-like pigment was observed in glia on light microscopy. Small amounts of electron-dense material were present on ultrastructural examination within oligodendroglia and astrocytes, but this material did not contain fingerprint-like profiles. It is difficult to be certain, as the authors discuss, that a diagnosis of OLD with pigmented glia is appropriate on the basis of so little pigment in a small biopsy. Because this diagnosis remains one of exclusion we suggest that it should only be advanced in the presence of typical histopathologic and ultrastructural findings in a biopsy containing both cerebral cortex and underlying white matter and in the appropriate clinical context.

There are few biochemical studies of this entity. One study ${ }^{14}$ recorded an excess of esterified cholesterol and a decrease in phospholipid, which were interpreted as reflecting active demyelination. In contrast, another ${ }^{7}$ did not detect a significant change in the amount of phospholipid or cholesterol esters, but did find alterations in the fatty acid profile; in particular decreased arachidonic and cervonic acids, and increased plasmalogen levels. In an unusual case of OLD with pigmented glia associated with globoid cells, there were by contrast markedly elevated levels of very long chain fatty acids ${ }^{8}$ which were interpreted as evidence for a peroxisomal defect. Whether these alterations represent an underlying abnormality in the regulation of lipid synthesis, or whether they are secondary to abnormal catabolism is unclear, but no single abnormality is common to all these cases.

The variable, if somewhat imprecisely documented, patterns of familial inheritance, the non-specific nature of the ultrastructural inclusions, as well as the inconstant histochemical features all argue that this diagnostic entity might not have a unitary pathogenic basis.

\section{ACKNOWLEDGEMENT}

The authors thank the technical staff of The Toronto Hospital Neuropathology Laboratory for their skilled assistance.

\section{REFERENCES}

1. Peiffer J. The pure leukodystrophic forms of orthochromatic leukodystrophies (simple type, pigment type). Handb Clin Neurol 1970; 10: 105-119.

2. Van Bogaert I, Nyssen R. Le type tardif de la leukodystrophie progreesive familiale. Rev Neurol (Paris) 1936; 65: 21-45.

3. Tunon T, Ferrer I, Gallego J, et al. Leukodystrophy with pigmented glial and scavenger cells (pigmentary type of orthochromatic leucodystrophy). Neuropathol Appl Neurobiol 1980; 14: 337344.

4. Seiser A, Jellinger K, Brainin B. Pigmentary type of orthochromatic leukodystrophy with early onset and protracted course. Neuropediatrics 1989; 21: 48-52.

5. Pietrini V, Tagliavini F, Pilleri G, Trabattoni CR, Lechi A. Orthochromatic leukodystrophy with pigmented glial cells: an adult case with clinical anatomical study. Acta Neurol Scand 1979; 59: 140-147.

6. Constantinidis J, Wisniewski TM. The dominant form of the pigmentary orthochromatic leukodystrophy. Acta Neuropathol 1991; 82: 483-487.

7. Gray F, Destee A, Bourre JM, et al. Pigmentary type of orthochromatic leukodystrophy (OLD); a new case with ultrastructural and biochemical study. J Neuropathol Exp Neurol 1987; 46(5): 585596.

8. Taniike M, Fujimara H, Kogaki S, et al. A case of pigmentary orthochromatic leukodystrophy with early onset and globoid cells. Acta Neuropathol 1992; 83: 427-433.

9. Belec L, Gray B, Louan F, et al. Leucodystrophie orthochromatique pigmentaire : maladie de Van Bogaert et Nyssan. Rev Neurol (Paris) 1988; 144: 347-357.

10. Okeda R, Matsuo T, Kawahara Y, et al. Adult pigment type (Peiffer) of sudanophilic leukodystrophy. Pathological and morphometrical studies on two autopsy cases of siblings. Acta Neuropathol 1989; 78: 533-542.

11. Sotrel A. On the dual nature and lack of specificity of intracytoplasmic inclusions in a case of adult onset leukodystrophy. J Neuropathol Exp Neurol 1988; 47(4): 490-491.

12. Hauw J, Escourolle R. Filamentous and multilamellated cytoplasmic inclusions in progressive multifocal leukoencephalopathy. Acta Neuropathol 1977; 537: 263-265.

13. Calandriello L, Matteucci C, Bertini E, et al. Biopsy diagnosis of adult onset orthochromatic leukodystrophy. Clinical and brain biopsy findings. Ital J Neurol Sci 1992; 13: 787-792.

14. Yokoi S. Histopathological and histochemical aspects of leucodystrophy in the Japanese. In: Folch-Pi $\mathbf{J}$ and Bauer $\mathrm{H}$, eds. Brain Lipids and Lipoproteins and the Leukodystrophies. Amsterdam: Elsevier, 1963: 153-160. 